\title{
Two-step Explicit Methods for the Numerical Integration of aSystem of Second Order Initial Value Problems
}

\section{Gul MN*and Habib M}

Department of Mathematics, University of Engineering and Technology, Lahore, Pakistan

\begin{abstract}
In this paper, authors present a new family of two-step explicit fourth order methods, for the numerical integration of second order periodic initial-value problems. These methods are explicit in nature and we intend to use them, in future, as a predictor for the family of direct hybrid methods. Their stability properties and the efficiency are also discussed. Considering some numerical results, authors saw that the new methods are superior to the existing explicit methods.
\end{abstract}

AMS subject classifications: 65L05

Keywords: Periodic initialvalue problem; Stability properties; Interval of periodicity; P-stable

\section{Introduction}

Authors are mainly concerned with the numerical integration of the special second order initial value systems of order $m$,

$$
y^{\prime \prime}=\frac{d^{2} y}{d t^{2}}=f(t, y), y(0), y^{\prime}(0) \text { given and } \mathrm{t}>0 \text {, }
$$

in which the first derivative does not appear explicitly. Such problems have periodic solutions and they are common in celestial mechanics, quantum mechanical scattering theory, theoretical physics and chemistry, and electronics [1,2]. The direct hybrid methods Cash [3] and Chawla [4] are implicit and when applied to a non-linear equation of the form (1), they need a predictor to start the iterations. These predictors should be explicit in nature Gladwell and Thomas [5] and Wang [6]

Author describes the basic theory of stability of symmetric multistep methods. Authors develop the explicit fourth order methods. Authors also consider the stability properties. Finally in Section 5, Authors give numerical examples which show that the new explicit methods are more efficient than the other well-known explicit methods, Chawla [7], [8], Chawla and Al-Zanaidi [9], and Franco [10].

\section{Basic Theory}

Lambert and Watson [11] introduce the scalar test equation

$$
y^{\prime \prime}=-\lambda^{2} y
$$

to investigate the stability properties of the methods for solving the initial value problem (1). When authors applied a symmetric twostep method to the scalar test equation (2) they obtained a difference equation of the form

$$
y_{n+1}-2 A(H) y_{n}+y_{n-1}=0
$$

where $\mathrm{H}=\lambda \mathrm{h}, \mathrm{h}$ is the step length, $A(H)=\frac{B(H)}{C(H)}$ where $\mathrm{C}(\mathrm{H})$ and $\mathrm{B}(\mathrm{H})$ are polynomials in $\mathrm{H}$ and $\mathrm{y}_{\mathrm{n}}$ is the computed approximation to $\mathrm{y}(\mathrm{nh}), \mathrm{n}=0,1,2, \ldots$. Following Coleman [12], when authors apply a symmetric two-step method to the scalar test equation (2) then authors have the difference equation (3). The characteristic equation associated with (3) is given by (4),

$$
r^{2}-2 A(H) r+1=0
$$

and the roots of the characteristic equation (4) are denoted by r1 and $\mathrm{r} 2$.

Definition 1: The numerical method (3) is said to have interval of periodicity, Lambert and Watson [11].

$$
\begin{aligned}
& \left(0, H_{0}^{2}, \text { if for all } H^{2} \in 0, H_{0}^{2}\right), \mathrm{r}_{1} \text { and } \mathrm{r}_{2} \text { satisfy } \\
& r_{1}=e^{i \theta(H)} \text { and } r_{2}=e^{-i \theta(H)}
\end{aligned}
$$

where $\theta(\mathrm{H})$ is a real function of $\mathrm{H}$.

Definition 2:The method (3) is P-stable if its interval of periodicity is $(0, \infty)$ Lambert and Watson [11].

Definition 3:The method (3) is unconditionally stable if $|r 1| \leq 1$ and $|r 2| \leq 1$ for all values of $\lambda \mathrm{h}$, Hairer [13].

Theorem 1: A method which has the characteristic equation (4), has an intervalof periodicity $\left(0, H_{0}^{2}\right)$, if, for all $H 2 \in\left(0, H_{0}^{2}\right),|A(H)|<1$.

Having stated the basic theory, authors developed the new explicit methods EXP4, Simos [14].

\section{New Explicit Method}

For the numerical integration of the second order initial value problem (1), consider the explicit fourth order method, which the authors will denote as EXP4 and is given by

$$
\begin{aligned}
& \bar{y}_{n+1}=2 y_{n}-y_{n-1}+h^{2} f_{n} \\
& \overline{\bar{y}}_{n+1}=2 y_{n}-y_{n-1}+h^{2}\left(A\left(\bar{f}_{n+1}+f_{n-1}\right)+B f_{n}\right) \\
& y_{n \pm \alpha}=G \pm y_{n+1}+H_{ \pm} y_{n}+I_{ \pm} y_{n}+h^{2}\left(j_{ \pm} \overline{\bar{f}}_{n+1}+K_{ \pm} f_{n}+L_{ \pm} f_{n-1}\right)
\end{aligned}
$$

*Corresponding author: Gul MN, Department of Mathematics, University of Engineering and Technology, Lahore, Pakistan, Tel: +(92)4299029210; E-mail: mgul@uet.edu.pk

Received January 15, 2016; Accepted January 29, 2016; Published February 03, 2016

Citation: Gul MN, Habib M (2016) Two-step Explicit Methods for the Numerical Integration of aSystem of Second Order Initial Value Problems. J Appl Computat Math 5: 286. doi:10.4172/2168-9679.1000286

Copyright: (๑) 2016 Gul MN, et al. This is an open-access article distributed under the terms of the Creative Commons Attribution License, which permits unrestricted use, distribution, and reproduction in any medium, provided the original author and source are credited. 


$$
\begin{aligned}
& y_{n+1}-2 y_{n}+y_{n-1}=h^{2}\left\{M\left(\overline{\bar{f}}_{n+1}+f_{n-1}\right)+N f_{n}+Q\left(f_{n+\alpha}+f_{n-\alpha}\right)\right\} \\
& f_{n \pm 1}=f\left(t_{n} \pm h, y\left(t_{n \pm 1}\right)\right), \bar{f}_{n+1}=f\left(t_{n}+h, \bar{y}\left(t_{n+1}\right)\right),
\end{aligned}
$$

with

$$
\overline{\bar{f}}_{n \pm 1}=f\left(t_{n} \pm h, \overline{\bar{y}}\left(t_{n \pm 1}\right)\right) \text {, and } f_{n \pm \alpha}=f\left(t_{n}+\alpha h, y\left(t_{n \pm \alpha}\right)\right)
$$

The method (6-9) is based on the hypotheses that authors put $\mathbf{f}_{\mathrm{n}}=\mathbf{f}\left(\mathrm{t}_{\mathrm{n}}, \mathbf{y}\left(\mathrm{t}_{\mathrm{n}}\right)\right)$ and they construct an $\mathrm{O}\left(\mathrm{h}^{4}\right)$ order approximation of $\mathbf{y}_{n+1}$. Authors improve this approximation of $\mathbf{y}_{n+1}$ by using $\mathrm{O}\left(\mathrm{h}^{4}\right)$ order approximation of $\mathbf{y}_{\mathrm{n}+1}$. Then, they obtain approximation to ${ }_{\mathrm{yn}+\alpha}$ which depend on the $\mathrm{O}\left(\mathrm{h}^{4}\right)$ order approximation to $\mathbf{y}_{\mathrm{n}+1}$ and are of order $\mathrm{O}\left(\mathrm{h}^{4}\right)$.

Now in order to find the local truncation error (LTE), we expand all the expressions of the method with respect to $h$ around the point $\mathbf{y}_{n}$, as a Taylor series and get

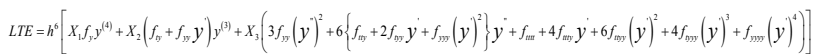

$$
\begin{aligned}
& \text { where } \\
& X_{1}=\frac{1}{360}-\frac{1}{12} A+Q\left\{A\left(\alpha^{2}-I_{+}-I_{-}\right)-\left(L_{+}+L_{-}\right)\right\} \\
& X_{2}=-\frac{1}{60}-2 \alpha Q\left(L_{-}-J_{-}\right) \\
& X_{2}=-\frac{1}{240}+\frac{1}{12} \alpha^{2} Q\left(1-\alpha^{2}\right)
\end{aligned}
$$

Here in (10), we have used subscripts to denoted the partial derivatives, for example

$$
f_{t y}=\sum_{i=1}^{m} \frac{\partial^{2} f}{\partial t \partial y_{i}}, f_{y y} y^{\prime}=\sum_{i=1}^{m} \sum_{i=1}^{m} \frac{\partial^{2} f}{\partial y_{i} \partial y_{i}} y^{\prime}, f_{t t y} y^{\prime}=\sum_{i=1}^{m} \frac{\partial^{4} f}{\partial t^{3} \partial y_{i}} y^{\prime}
$$

and so on. The parameters in the method (6-9) can be chosen freely. But to achieve fourth order accuracy, they must satisfy the conditions

$$
\begin{aligned}
& M=\frac{1}{12}-\frac{1}{12} \alpha^{2} Q, N=\frac{5}{6}+2 Q\left(\alpha^{2}-1\right), B=1-2 A, G_{+}=\alpha+I_{+}, G_{-}=-\alpha+I_{-}, \\
& J_{+}=L_{+}+L_{-}-J_{-}, H_{+}=1-\alpha-2 I_{+}, H_{-}=1+\alpha-2 I_{-}, \\
& K_{+}=\frac{1}{2} \alpha^{2}-\frac{1}{2} \alpha-I_{+}-2 L_{+}-L_{-}+J_{-}, K_{-}=\frac{1}{2} \alpha^{2}+\frac{1}{2} \alpha-I_{-}-L_{-}-J_{-} .
\end{aligned}
$$

So that the parametersa, Q, I, I, L, L, J are free. For simplicity, authors take them to be

$$
\alpha=\frac{1}{2}, A=\frac{1}{12}, Q=\frac{41}{90}, I_{+}=\frac{1}{4}, I_{-}=\frac{1}{4}, I_{-}=0, L_{+}=0, L_{-}=-\frac{1}{64}, J_{-}=\frac{3}{64}
$$

Hence, a particular EXP4 method is

$$
\begin{aligned}
& \bar{y}_{n+1}=2 y_{n}-y_{n-1}+h^{2} f_{n} \\
& \overline{\bar{y}}_{n+1}=2 y_{n}-y_{n-1}+\frac{1}{12} h^{2}\left(\bar{f}_{n+1}+10 f_{n}+f_{n-1}\right), \\
& y_{n+\frac{1}{2}}=\frac{1}{4}\left(3 \overline{\bar{y}}_{n+1}+y_{n-1}\right)-\frac{1}{16} h^{2}\left(\overline{\bar{f}}_{n+1}+5 f_{n}\right), \\
& y_{n-\frac{1}{2}}=-\frac{1}{2}\left(\overline{\bar{y}}_{n+1}-3 y_{n}\right)+\frac{1}{16} h^{2}\left(3 \overline{\bar{f}}_{n+1}+22 f_{n}-f_{n-1}\right),
\end{aligned}
$$

$y_{n+1}-2 y_{n}+y_{n-1}=\frac{1}{360} h^{2}\left\{-1\left(\overline{\bar{f}}_{n+1}+f_{n-1}\right)+54 f_{n}+164\left(f_{n+\frac{1}{2}}+f_{n-\frac{1}{2}}\right)\right\}$.

In the next section, we discuss the stability of EXP4.

\section{Stability analysis}

To analyze the stability, authors applied the methods (6-9) to the scalar test equation (2) and obtain (3) with

$$
\begin{aligned}
& \mathrm{C}(\mathrm{H})=1, \\
& B(H)=1-\frac{1}{2} H^{2}+\frac{1}{24} H^{4}-\frac{1}{2}\left[M A+Q\left\{A\left(I_{+}+I_{-}\right)+L_{+}+L_{-}\right\}\right] H^{6}+\frac{1}{2} Q A\left(L_{+}+L_{-}\right) H^{8} .
\end{aligned}
$$

Now to find the stability conditions, authors applied the Routh Hurwitz criterion which gives necessary and sufficient conditions for the zeros of a polynomial to have negative real parts to apply thiscriterion, we make the transformation $\mathrm{r}=(1+\mathrm{Z}) /(1-\mathrm{Z})$, which maps the circle $|r|=1$ into the imaginaryaxis $\operatorname{Re}(\mathrm{z})=0$, the interior of the unit circle into the half plane $\operatorname{Re}(\mathrm{z})<0$ and the point $\mathrm{r}=1$ into $\mathrm{z}=0$ Lambert [15]. So, authors have, from equation (4).

$$
(2-A(H)) z^{2}+(2+A(H))=0 \text {. }
$$

Hence the necessary and sufficient conditions for the stability of the methods (6-9) are

$$
\begin{aligned}
0 \leq 4-H^{2}+\frac{1}{12} H^{4}-\left[M A+Q\left\{A\left(I_{+}+I_{-}\right)+L_{+}+L_{-}\right\}\right] H^{6}+Q A\left(L_{+}+L_{-}\right) H^{8}, & (18) \\
& 0 \leq 1-\frac{1}{12} H^{2}+\left[M A+Q\left\{A\left(I_{+}+I_{-}\right)+L_{+}+L_{-}\right\}\right] H^{4}-Q A\left(L_{+}+L_{-}\right) H^{6} .
\end{aligned}
$$

When the parameters are given by (12), then the stability conditions (18) give

$$
\begin{aligned}
& 4-H^{2}+\frac{1}{12} H^{4}+\frac{1}{5760} H^{6}+\frac{41}{69120} H^{8} \geq 0, \\
& 1-\frac{1}{12} H^{2}-\frac{1}{5760} H^{4}+\frac{41}{69120} H^{6} \geq 0 .
\end{aligned}
$$

Also, it can be easily seen that the stability interval is $\mathrm{H} \in(0,2.55)$. In the next section authors consider a numerical example which shows the method EXP4 performs better than the methods in literature.

\section{Numerical Examples}

Let us consider the numerical solution of a linear problem, considered by Stiefel and Bettis[16], Lambert and Watson [11] and Cash [3],

$$
z^{\prime \prime}(t)+z(t)=0.001 e^{i t}, z(0)=1, z^{\prime}(0)=0.9995 i, z(t) \in C \text {. }
$$

This linear problem has the analytic solution

$\mathrm{Z}(\mathrm{t})=\mathrm{u}(\mathrm{t})+\mathrm{iv}(\mathrm{t}), \mathrm{u}(\mathrm{t}), \mathrm{v}(\mathrm{t}) \in \mathrm{R}$

where

$\mathrm{u}(\mathrm{t})=\cos (\mathrm{t})+0.0005 \mathrm{t} \sin (\mathrm{t})$,

$v(t)=\sin (t)-0.0005 t \cos (t)$

The analytic solution (21) represents motion on a perturbed circular orbit in the complex z-plane, as shown below in Figure 1.

The distance of the circular orbit from the origin at time $t$ is given by

$$
|z(t)|=\sqrt{(u(t))^{2}+(v(t))^{2}}
$$




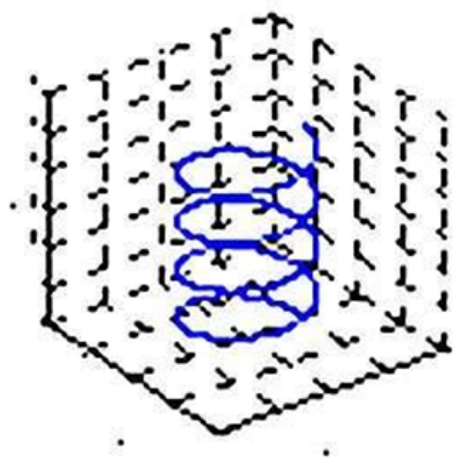

Figure 1: Spiral behaviour of $z(t)$.

$$
=\sqrt{1+(0.0005)^{2}}
$$

Following Lambert and Watson [11], authors write the complex scalar problem $(20)$ in the equivalent form for $\mathrm{u}(\mathrm{t})$ and $\mathrm{v}(\mathrm{t})$ as

$$
\begin{aligned}
& u^{\prime \prime}(t)+u(t)=0.001 \cos (t), u(0)=1, u^{\prime}(0)=0, u(t) \in R, \\
& v^{\prime \prime}(t)+v(t)=0.001 \sin (t), v(0)=1, v^{\prime}(0)=0.9995, v(t) \in R
\end{aligned}
$$

The solution to this pair of uncoupled, real, scalar linear problems is computed using the initial conditions at $\mathrm{t}=0$ and the analytic solution at $\mathrm{t}=\mathrm{h}$, that is we set

$$
\begin{aligned}
& u_{0}=1, u_{1}=\cos (h)+0.0005 h \sin (h), \\
& v_{0}=0, v_{1}=\sin (h)-0.0005 h \cos (h) .
\end{aligned}
$$

Authors obtained the global error at $t=40 \pi$ by comparing the solution obtained by the method $\backslash \mathrm{QTR}$ \{group\}\{EXP4\} with the analytic solution. Table 1 gives the numerical solutionproduced by the methods (1-4) for $|z(40 \pi)|=1.00197213$, obtained with step sizes $h=\pi / 2, \pi / 5, \pi / 6$, $\pi / 9$ and $\pi / 12$ respectively.

Where the methods are:

Method 1: Numerov made explicit by Chawla[7].

Method 2: A two stage fourth order "almost" P-stable by Chawla and Al-Zanaidi[9]. Method 3: An explicit hybrid method of Numerov by Franco [10].

Method 4: Two-step EXP4 method developed in this paper.

\begin{tabular}{|c|c|c|c|c|}
\hline $\boldsymbol{h}$ & Method 1 & Method 2 & Method 3 & Method 4 \\
\hline$\pi / 2$ & 1.00306 & 1.002003 & 1.001970 & 1.001971 \\
\hline$\pi / 5$ & 1.002217 & 1.001981 & 1.001971 & 1.001972 \\
\hline$\pi / 6$ & 1.002047 & 1.001975 & 1.001972 & 1.001972 \\
\hline$\pi / 9$ & 1.001978 & 1.001972 & 1.001972 & 1.001972 \\
\hline$\pi / 12$ & 1.001973 & 1.001972 & 1.001972 & 1.001972 \\
\hline
\end{tabular}

Table 1:Comparison of the approximations obtained by Methods 1-4 for $|z(40 \pi)|$.

\begin{tabular}{|c|c|c|c|c|}
\hline$h$ & Method 1 & Method 2 & Method 3 & Method 4 \\
\hline$\pi / 4$ & $0.101 \times 10^{-2}$ & $0.00314 \times 10^{-2}$ & $0.000219 \times 10^{-2}$ & $0.000100 \times 10^{-2}$ \\
\hline$\pi / 8$ & $0.123 \times 10^{-4}$ & $0.00507 \times 10^{-4}$ & $0.000245 \times 10^{-4}$ & $0.000134 \times 10^{-4}$ \\
\hline$\pi / 16$ & $0.180 \times 10^{-6}$ & $0.00823 \times 10^{-6}$ & $0.000170 \times 10^{-6}$ & $0.00020 \times 10^{-6}$ \\
\hline
\end{tabular}

Table 2:Comparison of the absolute errors in the approximations obtained by Methods $1-4$ for $|z(40 \pi)|$.
On comparing these results, we see that for this problem and for these step sizes, method 4 is more accurate than any of the other methods, especially for larger step sizes. In Table 2, below, authors also present, for comparison purposes, the absolute errors in the computed values of $z(40 \pi) \mid$ produced byusing these methods with step sizes $\mathrm{h}=\pi / 4, \pi / 8$, and $\pi / 16$ respectively.

Table 2 also shows that as the authors halve the step size then the absolute error decreases by a factor of approximately $2^{4}$. Finally, authors present some conclusions in the next section.

\section{Conclusions}

Authors have developed explicit fourth order method, which we will be using as a predictor for a class of direct hybrid methods. From the Tables 1 and 2, above, we conclude that the method EXP4 performs much better than the existing methods in the literature and they converge rapidly as compared with other methods. Since the methods based on Numerov usually involve evaluation of first order derivative, which does not appear in the class of problems given byequ(1). So, the methods in literature have a big disadvantage, while our methods EXP4 integrate the equation directly, without requiring the need to calculate the first order derivative.

\section{References}

1. Landau LD, Lifshit FM (1965) Quantum Mechanics.Peramon Press, New York

2. Liboff RL (1980) Introductory Quantum Mechanics.Addison Wesely, Reading, MA

3. Cash JR (1981) High order P-stable formulae for the numerical integration of periodic initialvalue problems. Numer Math37: 355-370.

4. Chawla MM (1981) Two-step fourth order P-stable methods for second order differentialequation. BIT21: 190-193.

5. Gladwell I, Thomas RM (1990) Efficiency of methods for second order problems. IMA J Numer Anal 10: 181-207.

6. Wang J (1993) Numerical methods for Hamiltonian system. Southern Methodis University, Dallas Texas.

7. Chawla MM (1984) Numerov made explicit has better stability. BIT 24: 117-118.

8. Chawla MM (1986) A new class of explicit two-step fourth order methods for $y=f(x, y)$ withextended intervals of periodicity. $J$ of Compt and Appl Math14 467-470.

9. Chawla MM, Al-Zanaidi MA (1989) A two-stage fourth order "almost" P-stable methodfor oscillatory problems. J of Compt and Appl Math89: 115-118.

10. Franko JM (1995) An explicit method of Numerov type for second-order periodic initial-valueproblems. J of Compt and Appl Math59: 79-90.

11. Lambert JD, Watson IA (1976) Symmetric multistep methods for periodic initialvalueproblems. J InstMathsApplics18: 189-202.

12. Coleman JP (1989) Numerical Methods for $y=f(x, y)$ via rational approximation for thecosine. IMA J Numer Anal9: 145-165.

13. Hairer $E$ (1979) Unconditionally stable methods for the second order differential equations.Numer Math 32: 373-379.

14. Simos TE, Mousadis G (1994) Some new Numerove-type methods with minimum phase-lag for the numerical integration of the radial Schrodinger equation.Comput Math Appl83: 1145-1153.

15. Lambert DJ (1973) Computational Methods in Ordinary Differential Equations John Wiley and Sons.

16. Stiefel E, Bettis DG (1969) Stabilization of Cowell's Method.Numer Math 13 154-175. 